\title{
Búsqueda de bacterias con actividad EC 3.2.1.4 (endo-1,4- beta-glucanasa) en Eisenia foetida (Oligochaeta, Lumbricidae)
}

\author{
Carmen Amelia Salvador, ${ }^{1,2}$, Maira Rojas ${ }^{2}$, Gabriela Jaramillo-Kouperman², \\ Lucía Yépez ${ }^{1}$, Juan Pablo Suárez ${ }^{3}$, Leyanis Mesa ${ }^{4}$ y César Paz-y-Miño ${ }^{2}$ \\ ${ }^{1}$ Centro Universitario de Investigación Científica y Tecnológica. Universidad Técnica del Norte (UTN). \\ Ibarra, Ecuador. pochasalvador@yahoo.com. ${ }^{2}$ Instituto de Investigaciones Biomédicas, \\ Universidad de las Américas (UDLA). Quito, Ecuador. ${ }^{3}$ Centros de Investigación, Transferencia de Tecnología, \\ Extensión y Servicios. Universidad Particular de Loja (UTPL). Loja, Ecuador. ${ }^{4}$ Centro de Análisis de Procesos, \\ Universidad Central "Marta Abreu" de Las Villas. Cuba.
}

Recibido: 16, 03, 2012; aceptado: 05, 10, 2012

\begin{abstract}
RESUMEN.- La lombriz de tierra, Eisenia foetida, es hospedero de numerosos microorganismos simbiontes, muchos de los cuales han sido identificados recientemente, así como se ha descrito su actividad enzimática. Estos microorganismos suelen presentar actividad enzimática de diferente tipo. Algunos podrían presentar interés industrial por sus potenciales aplicaciones. En el presente trabajo, se eligió identificar aquellos microorganismos que tienen actividad endo- $\beta$-1,4-glucanasa, y evaluar dicha actividad por el método de los azúcares reductores de Nelson-Somogyi. Dichos azúcares reductores fueron liberados después de la incubación de las bacterias con la solución de carboximetilcelulosa (CMC). Las colonias aisladas de interés fueron cultivadas en Plate Count Agar, PCA (Difco), y su identificación se llevó a cabo mediante el análisis de la región 16SDNAr. Comparando los resultados con la base de datos NCBI, se encontró que las especies productoras de la actividad endo- $\beta$-1,4-glucanasa fueron: Burkholderia fhytofirmans, Bacillus amyloliquefaciens, Bacillus licheniformis, y Acidovorax delafieldii. Los aislamientos produjeron un porcentaje de degradación interesante en relación al control positivo empleado. Se presentan comparaciones de la degradación causada por las diferentes colonias aisladas.
\end{abstract}

PALABRAS CLAVE: Endo- $\beta$-1,4-glucanasa, enzimas, lombriz de tierra, microbiota, 16SDNAr.

\begin{abstract}
The earth worm, Eisenia foetida, is host for numerous symbiotic microorganisms, many of which have been recently identified, as well as the description of their enzymatic activity. These microorganisms usually present enzymatic activity of different type, and some may present industrial interest due to their potential applications. In the present research, it was decided to identify those microorganisms that present endo- $\beta$-1,4-glucanasa activity, and to evaluate this activity by the Nelson-Somogyi method of reducing sugars. Reducing sugars were released after the incubation of the bacteria colonies with carboxymethyl cellulose (CMC) solution. The isolated colonies of interest were cultured in laboratory and their identification was made through analysis of the 16S DNAr region. By comparing the results with the NCBI database, it was found that the endo- $\beta-1,4-$ glucanase activity producing species were: Burkholderia fhytofirmans, Bacillus amyloliquefaciens, Bacillus licheniformis, and Acidovorax delafieldii. The isolations produced an interesting degradation percentage in relation to the positive control utilized. Comparisons of the degradation caused by the different isolated colonies are presented.
\end{abstract}

KEYWORDS: endo- $\beta$-1,4-glucanase, earth worm, enzymes, microbiota, 16SDNAr. 


\section{INTRODUCCIÓN}

La mayor fracción de materia orgánica en los ecosistemas terrestres está constituida por celulosa. Los microorganismos del suelo son los principales responsables de transferir el carbono de la celulosa y otros compuestos a la atmósfera, pero su contribución no es exactamente conocida (Haichar, 2007); algunos de estos microorganismos viven en simbiosis en la lombriz de tierra, que también está ligada a la materia en descomposición (Edwards y Bohlen, 1996). Los microorganismos que habitan en el suelo ingresan al tubo digestivo de la lombriz de tierra para servir de alimento, brindar un componente energético a la dieta de la lombriz y para activar o desactivar una producción enzimática (Zirbes, 2007).

La población microbiana ingerida tiene entre sus funciones la de ayudar a digerir la materia orgánica del suelo (Brown y Lavelle, 2000) y desencadenar una actividad enzimática (Aira et al., 2006), estas comunidades microbianas muestran una asociación oportunista (Curry y Schmidt, 2006). En Eisenia foetida también se han descrito mecanismos de activación de la producción de actividades enzimáticas, encontrándose las actividades, celobiasa y endoglucanasa, relacionadas con las actividades celulolíticas (Prabha, 2007). Las celulasas descomponen los restos vegetales de los que se alimenta la lombriz de tierra, pueden participar en la descomposición de lignina y en el proceso de humificación (Brown y Lavelle, 2000). La actividad celulolítica puede ser propia de la especie o estar relacionada con la flora microbiana ingerida a partir del suelo, para permitir la hidrólisis de sustratos como la celulosa y el manano (Lattaud et al., 1997).

Estas enzimas facilitan el reciclaje de los elementos nutritivos a partir de materia orgánica y su conversión en formas fácilmente absorbibles por las plantas (Edwards y Bohlen, 1996). Para la hidrólisis y el metabolismo de la celulosa los microorganismos producen celulasas extracelulares libres o asociadas a la célula. Las endoglucanasas EC 3.2.1.4 actúan en las porciones amorfas de las fibras de celulosa (Ramirez y Coha, 2003). La actividad celulolítica ha sido encontrada en algunos microorganismos pertenecientes a los Phylum Firmicutes, Actinobacteria, Fibrobacteres, Bacteroidetes, así como Proteobacteria. Entre las bacterias celulolíticas más abundantes se puede citar Cellulomonas sp, Microbisporabispora $\mathrm{sp}$, Thermomonospora sp, Cytophaga sp, Corynebacterium sp, Vibrio sp, Bacillus sp, Pseudomonas sp y Thermobifida sp (Gaitan y Pérez, 2007). De estas algunas especies como Clostridium thermocellum cumplen con el proceso de hidrólisis de la celulosa de manera eficiente pues poseen un complejo enzimático que favorece la celulolisis, constituido de endo $\beta$ 1-4 glucanasa, exo $\beta$ 1-4 glucanasa y $\beta$ - glucosidasa, este complejo puede ubicarse en una estructura denominada celulosoma (Mitsuhiro, 2010). Algunas colonias aisladas de Burkholderia fhytofirmans y Bacillus sp., producen celulasas poco comunes (Sessitsch, 2005), que tienen alta actividad y termoestabilidad a un alto rango de $\mathrm{pH}$ (Miranda, 2009).

Los organismos más comunes de esta lombriz son los bacilos Gram positivos (Vinceslas-Akpa y Loquet, 1995) del género $B a$ cillus. Sin embargo, también se han encontrado microorganismos Gram negativos a nivel de los órganos excretores de la lombriz llamados nefridios. Análisis de las bacterias ligadas a los nefridios han mostrado que cada especie de lombriz alberga una especie bacteriana distinta y que las especies de bacterias forman un haz monofilético en el género Acidovorax, pareciendo ser que hay una asociación específica, resultado de un ancestro bacteriano común (Davidson y Stahl, 2006).

En el presente estudio se desea hallar en los intestinos de la lombriz bacterias productoras de actividades endo-1,4-beta-glucanasa y comprender si entre las especies encontradas existen diferencias en la degradación de la celulosa. 


\section{MATERIALES Y MÉTODOS}

Recolección de lombrices.- Los individuos provinieron del Vivero del Programa Quito-Verde del Municipio de Quito, estos fueron mantenidos en el laboratorio del Instituto de Investigaciones Biomédicas, IIB de la Universidad de las Américas. Las lombrices fueron colocadas en una caja rectangular de plástico con una capacidad de 101 en un suelo orgánico húmedo con una temperatura entre $15^{\circ} \mathrm{C} \mathrm{y} 25^{\circ} \mathrm{C}$. El sustrato en el que fueron conservadas se compone de tierra de superficie y desechos vegetales en proporciones de $2: 1$, respectivamente.

Disección de lombrices de tierra y recolección del tubo digestivo.- Las lombrices de tierra fueron lavadas con agua destilada, sumergidas en etanol al $45 \%$ durante $5 \mathrm{~s}$, colocadas en un congelador durante 3 min para que el agua del tejido epidérmico de la lombriz se encuentre cristalizado y se pueda proceder fácilmente con la disección. Se extrajo el tubo digestivo de la lombriz y se lo colocó en $1 \mathrm{ml}$ de agua destilada estéril. La mezcla que contiene extractos de tubo digestivo no filtrados, se colocó en el vortex (Genius) durante $15 \mathrm{~s}$, a velocidad mediana. Esta mezcla se colocó en tres diluciones sucesivas de $1 \mathrm{ml}$ de agua destilada, con el objetivo de obtener concentraciones en diluciones de base diez.

\section{Detección de la actividad enzimática en} los extractos filtrados y no filtrados.- Después de la disección se tomó el tubo digestivo. Éste fue colocado en $1 \mathrm{ml}$ de agua destilada, para ser homogeneizado con ayuda del vortex (Genius); esta mezcla corresponde a los extractos no filtrados, y se refiere a los diferentes tejidos del tubo digestivo que contienen microorganismos y estructuras como el celulosoma. En cambio los extractos filtrados son obtenidos mediante la filtración en una membrana Millipore $0.45 \mu \mathrm{m}$ (Merck) de la mezcla del tubo digestivo en agua destilada; este filtrado corresponde al segundo extracto y contiene en su gran mayoría moléculas como enzimas. Ambos extractos sirven de guía para determinar el origen, ya sea microbiano o propio de la lombriz, de la actividad EC 3.2.1.4 endo-1,4- beta-glucanasa.

El origen de la actividad se determina colocando los extractos anteriores, en tubos de vidrio de $20 \mathrm{ml}$, que contienen $10 \mathrm{ml}$ de agua destilada, $0.9 \mathrm{~g}$ de gelatina y $0.2 \mathrm{~g}$ de carboximetilcelulosa. Para verificar que no existe presencia de azúcares reductores en la gelatina natural, se empleó el método de licor de Fehling Causse-Bonnans (Isla, 2011) pues estos pueden registrar resultados falsos el test enzimático de Nelson-Somogyi. No se encontraron azúcares reductores. Este sustrato de CMC y gelatina fue previamente autoclavado a $120^{\circ}$ C para posteriormente inocular los extractos filtrados y no filtrados.

Después de 15 horas de la inoculación de ambos extractos se procedió al empleo del protocolo de Nelson-Somogy (Nelson, 1944) para el análisis de la glucosa obtenida producto de la degradación de la celulosa y para verificación de la presencia de las enzimas en alguno de los extractos. Se colocó un control positivo con una enzima comercial celulasa de Aspergillus niger C1184-5KU Sigma-Aldrich; para la preparación de la enzima se colocó 0.1 g de la enzima comercial en $10 \mathrm{ml}$ de agua destilada. El control negativo contenía agua destilada estéril en lugar de la solución bacteriana o enzimática.

Este ensayo es realizado para asegurarnos de la presencia de microbiota simbionte productora de la actividad enzimática en los extractos del tubo digestivo, previo al ensayo de aislamiento.

Aislamiento, purificación, selección y preparación de las colonias.- Se sembró $40 \mu 1$ del extracto no filtrado en medio Plate Count Agar, PCA (Difco), para realizar el contaje de las colonias. Las colonias que crecieron fueron aisladas, purificadas, sembradas nuevamente en caldo nutritivo (Difco) a $37^{\circ}$ 
$\mathrm{C} \pm 1$ por 24 horas. Luego de la incubación se aislaron colonias en medio PCA, para confirmar la pureza. Los aislamientos madre fueron conservados en criotubos (Fisher) con esferas de plástico a $-80^{\circ} \mathrm{C}$.

\section{Detección de la actividad celulolítica EC} 3.2.1.4 (endo-1,4- beta-glucanasa) por el método de azúcares reductores de Nelson-Somogy.- Esta prueba se realizó con las bacterias aisladas de los extractos no filtrados, sin presencia de gelatina como soporte, siguiendo el método de azúcares reductores, que consistió en preparar el sustrato diluido de carboximetilcelulosa (CMC) al $2 \%$. De esta preparación se tomó $1 \mathrm{~g}$ de este sustrato en $50 \mathrm{ml}$ de tampón fosfato de sodio ( $\mathrm{pH}$ 5.8). Esta mezcla se homogeneizó rápidamente durante $5 \mathrm{~min}$ con la ayuda de un agitador magnético para evitar cualquier tipo de agregaciones, para luego enfriar a temperatura ambiente. Se emplearon los mismos controles para la detección de la actividad enzimática en los extractos filtrados y no filtrados.

\section{Preparación y lectura de las muestras.-}

Se colocó $0.5 \mathrm{ml}$ de bacterias previamente preparadas en agua destilada estéril a escala Mc. Farland 2 en un tubo que contenía $1 \mathrm{ml}$ de sustrato diluido del medio CMC. Se dejó a baño María durante $15 \mathrm{~min}$, se procedió según el protocolo de Nelson-Somogy (Nelson, 1944).

Análisis por espectrofotometría.- Se sacaron los tubos del baño María y se colocan $3 \mathrm{ml}$ de solución Acido dinitrosalicílico (DNS) en cada tubo. Se colocan todos los tubos en un baño María a $100^{\circ} \mathrm{C}$ durante $5 \mathrm{~min}$. Luego se enfriaron los tubos y se midió la cantidad de glucosa producida por la degradación de la celulosa en el UVmini-1240-Shimadzu Spectrophotometer a $550 \mathrm{~nm}$.

Para interpretar los resultados de la degradación producida por las colonias bacterianas, se aplicó la prueba de Kruskal-Wallis (Chan y Walmsley, 1997), para esto se uso el programa Minitab 15(Win2000/XP/2003/Vista). Se planteo la hipótesis nula $(\mathrm{H} 0)$ en la que no existe diferencia estadística en la degradación de la celulosa generada por las bacterias y la hipótesis alternativa (H1) en la que existe diferencia estadística en la degradación de la celulosa generada por las bacterias.

\section{Secuenciamiento e Identificación mo-} lecular.- El ADN fue extraído de aproximadamente $2 \times 10{ }^{9}$ bacterias (en $1 \mathrm{ml}$ de agua destilada) que se encontraban en caldo nutritivo (Difco) empleando un kit de purificación PureLink Genomic DNA (Invitrogen). Un volumen final de $50 \mu 1$ por reacción fue amplificado en un termociclador Thermal cycler Multigene Gradient (Labnet International, Inc.). Por reacción se tuvo $10 \mu \mathrm{l}$ de DNA a una concentración de $5 \mathrm{ng} / \mu \mathrm{l}, 1 \mu \mathrm{M}$ de cada primer, $0.20 \mathrm{mM}$ de dNTPs, de $1.5 \mathrm{mM}$ de $\mathrm{MgCl} 2$ y $1.25 \mathrm{U}$ Taq Polimerasa (Invitrogen). Los primers empleados para la secuenciación de la región 16SDNAr son 8F AGAGTTTGATCCTGGCTCAG Universal Turner (Turner, 1999) y 1492R (s) GGTTACCTTGTTACGACTT Universal Lane (Lane, 1991).

Los fragmentos de amplificación fueron verificados por electroforesis en geles de agarosa al $1 \%$. Se purificó la PCR con el PureLink PCR Purification Kit (Invitrogen). Las secuencias Forward y Reverse fueron alineadas usando el programa Bioedit (Harrison y Langdale, 2006). Las secuencias presentes en los organismos fueron alineadas a organismos presentes en el Database GenBank database (Septiembre 2011), usando el Basic Local Alignment Search Tool(Blast) del National Center for Biotechnlogy Information (http://blast.ncbi.nlm.nih.gov/ Blast.cgi?PROGRAM=blastn\&BLAST_PROGRAMS $=$ megaBlast\&PAGE_TYPE $=$ Blast Search\&SHOW_DEFAULTS $=$ on\&LINK_LO$\mathrm{C}=$ blasthome.

\section{RESULTADOS}

En el tubo digestivo de los siete individuos de Eisenia foetida se encontró una media aritmética de $2.07 \times 10^{5} \mathrm{CFU} / \mathrm{g}$ de bacterias totales. De esta microbiota se seleccionaron cinco aislamientos, que produjeron después 


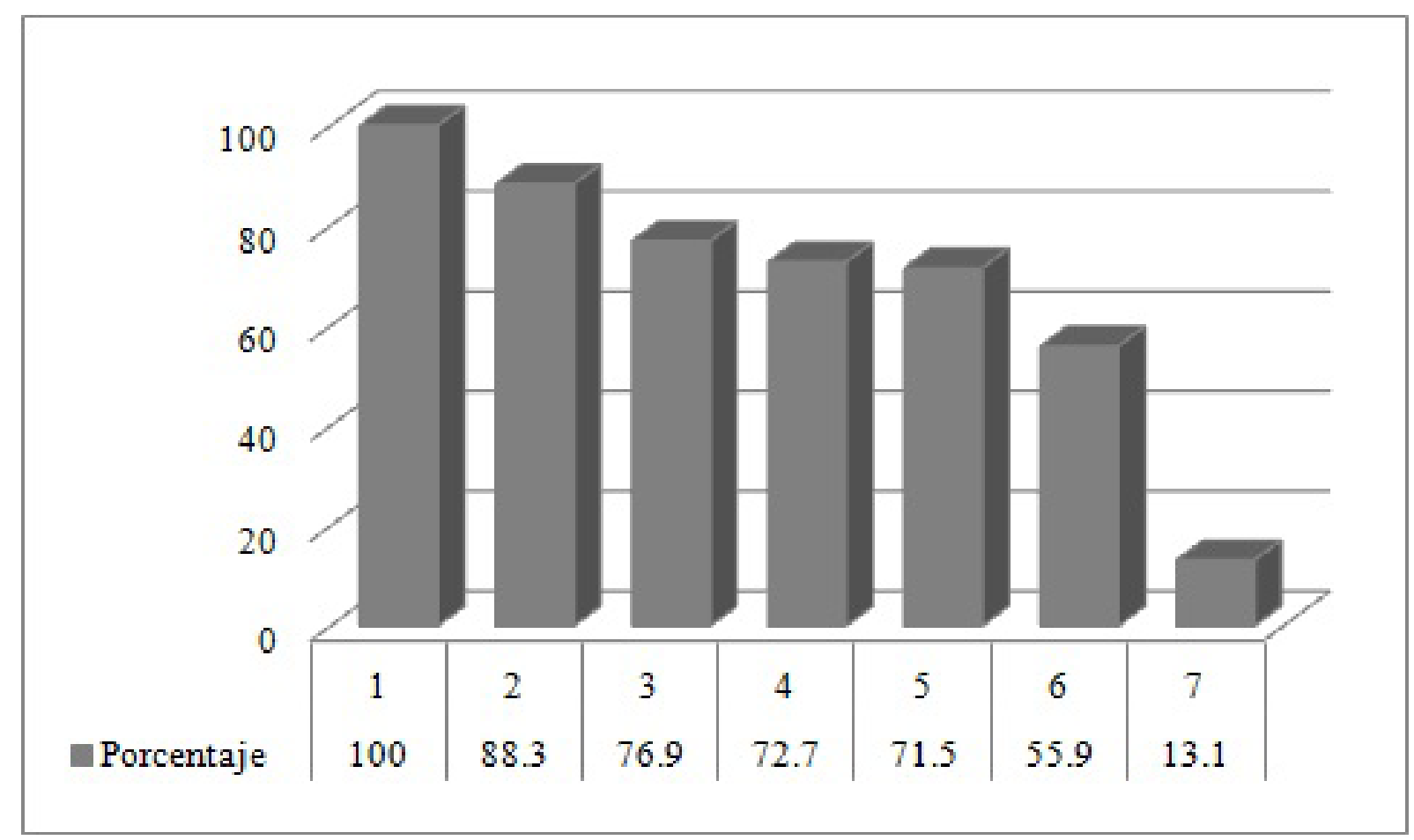

Especies: Aspergillus niger C1184-5KU-Control + (1), Bacillus amyloliquefasciens (2), Acidovorax delafieldii (3), Burkholderia phytofirmans (4), Bacillus amyloliquefasciens (5), Bacillus licheniformis (6), Control - (7)

Figura 1. Porcentaje de glucosa después de la degradación de la carboximetilcelulosa.

del tiempo de incubación, una absorbancia de más de uno, esta medida fue tomada debido a que la Absorbancia, Ab que presentó la enzima comercial superaba este valor en cada una de las mediciones realizadas.

Después de la alineación de las secuencias en el Blast, las cinco especies tienen secuencias similares en un $99 \%$ a Burkholderia fhytofirmans (CL1), Bacillus amyloliquefaciens (CL5) (CL9), Bacillus licheniformis (CL16), y a Acidovorax delafieldii (CL8).

En relación a la presencia de glucosa promovida por la degradación bacteriana de la celulosa en presencia de la actividad EC 3.2.1.4 (endo-1,4-beta-glucanasa, ésta fue transformada en porcentaje como se observa en la Figura 1.

Solo se encontraron actividades enzimáticas celulolíticas de origen posiblemente microbiano en los extractos no filtrados, sin embargo en otros estudios (Edwards y Bohlen,
1996), se han encontrado en los extractos no filtrados, enzimas celulolíticas secretadas por la propia lombriz de tierra y no por la microbiota simbionte. A nivel cualitativo se presenció un cambio en la constitución de la mezcla pues se formó un halo vidrioso-líquido que nos indicaba la degradación de la actividad endo-1,4- beta-glucanasa.

Del extracto digestivo no filtrado se seleccionaron las cinco bacterias para la determinación cuantitativa de la actividad endo-1.4-beta-glucanasa. Estas bacterias, bajo las condiciones descritas, degradaron de manera similar los sustratos de CMC, pues la prueba de Kruskal-Wallis proyectó el valor de diez y ocho para los datos de absorbancia medidos; para esto previamente se encontró que el valor de ji-cuadrada para 4 grados de libertad es 9.48 para un nivel de confianza del $95 \%$, en la prueba ANOVA el valor es de 1.64 y la probabilidad es del 0.82 . Tabla 1 . 
Tabla 1. Análisis estadístico empleado para medir la degradación de la carboximetilcelulosa.

\begin{tabular}{lcccc}
\hline \multicolumn{2}{l}{ Prueba de Kruskal-Wallis } & & \\
\hline Grupo & $\mathbf{N}$ & Mediana del promedio & $\mathbf{Z}$ \\
\hline $\mathrm{Cl} 1$ & 7 & 1.055 & 17.9 & -0.02 \\
$\mathrm{Cl} 5$ & 7 & 2.45 & 20.5 & 0.72 \\
$\mathrm{Cl} 8$ & 7 & 2.2 & 20.1 & 0.62 \\
$\mathrm{Cl} 9$ & 7 & 1.87 & 17 & -0.29 \\
$\mathrm{Cl16}$ & 7 & 1.54 & 14.4 & -1.03 \\
General 35 & & & 18 & \\
$\mathrm{H}=1.64$ & $\mathrm{G}=4$ & $\mathbf{P}=\mathbf{0 . 8 0 2}$ & & \\
$\mathrm{H}=1.64$ & $\mathrm{G}=4$ & $\mathbf{P}=\mathbf{0 . 8 0 1}$ & & \\
\hline
\end{tabular}

Parece ser que esta actividad celulolítica puede originarse de los microorganismos ingeridos en la dieta nutricional de la lombriz o en algunos casos estar fijada a partículas del sistema digestivo de Eisenia foetida, como el celulosoma.

\section{DISCUSIÓN}

El número de unidades formadoras de colonias (UFC) que se encontró con esta especie coincide con otros estudios realizados (Vinceslas-Akpa y Loquet, 1995), se esperaba encontrar una abundancia bacteriana más alta en los intestinos de Eisenia foetida, pues la microbiota investigada proviene del suelo y de materiales orgánicos del componente edáfico ecuatoriano que es considerado megadiverso (Estrella, 2005). También otros estudios han mostrado que la diversidad microbiana total de la especie podría ser independiente del sitio en el que se colectó la lombriz (Salvador et al., 2011), porque posiblemente Eisenia foetida posee un mecanismo de selección de las especies microbianas que ingiere (Zirbes, 2007).

Con el valor de la probabilidad de 0.82 en la prueba de Kruskal-Wallis (Chan y Walmsley, 1997) se concluye que no hay variación entre las colonias aisladas cuando se trata de degradar la carboximetilcelulosa. Al respecto se conoce que los géneros Acidovorax, Burkhol- deria y Bacillus, posiblemente requieren de mecanismos que estimulen la actividad enzimática como la respuesta fisiológica físico-química de la lombriz de tierra como hospedero (Brown y Lavelle, 2000). Se conoce además que los géneros Burkholderia, Bacillus y Acidovorax comprenden algunas especies que son transitorias, que se encuentran comúnmente en el suelo y que posiblemente son estimuladas por condiciones ambientales del componente edáfico. De los géneros encontrados, Acidovorax ha sido ampliamente estudiado porque puede ser heredado (Davidson y Stahl, 2006); la transmisión de bacterias simbiontes en las cápsulas de los huevos ha sido recientemente demostrada en Eisenia foetida, el género incluye especies que han sido aisladas del suelo y del fango (Heylen et al., 2008); sobre el origen de las bacterias se ha sugerido que el morfotipo de bacterias filamentosas, debe ser interpretada como una microfauna autóctona del tubo digestivo (Nelson, 1944), sin embargo, el origen de la microbiota sigue siendo controversial (Curry y Schmidt, 2006).

\section{AGRADECIMIENTOS}

A José Ignacio Laquidáin por el trabajo de traducción en inglés, la revisión del texto en español y las sugerencias brindadas. A Marthe Vinceslas-Akpa y Ricardo Oliva por los artículos científicos facilitados. 


\section{REFERENCIAS BIBLIOGRÁFICAS}

Aira M, Monroy F y Domínguez J. 2006. Eisenia foetida (Oligochaeta, Lumbricidae) activates fungal growth, triggering cellulose decomposition during vermicomposting. Microbial Ecology, 52(4): 738-747.

Brown G, Barois I y Lavelle, P. 2000. Regulation of soil organic matter dynamics and microbial activitity in the drilosphere and the role of interactions with other edaphic functional domains. European Journal of Soil Biology, 36(1): 177-198.

Chan Y y Walmsley R. 1997. Learning and Understanding the Kruskal-Wallis OneWay Analysis-of-Variance-by-Ranks Test for Differences Among Three or More Independent Groups. R. Physical Therapy, 77(12): 1755-176.

Curry J P y Schmidt O. 2006. The feeding ecology of earthworms. Pedology, 50(6):4463-477.

Davidson S K y Stahl D A. 2006. Transmission of Nephridial Bacteria of the Earthworm Eisenia fetida. Applied and Environmental Microbiology, 72(1): 769-775.

Edwards C A y Bohlen P J. 1996. The Biology and Ecology of Earthworms. $3^{\text {rd }}$ edition. Chapman and Hall. London, New York, $426 \mathrm{pp}$.

Estrella J, Manosalvas R, Mariaca J y Ribadeneria M. 2005. Biodiversidad y Recursos genéticos: Una guía para su uso y acceso en el Ecuador. Abya Yala. Quito-Ecuador. $27 \mathrm{pp}$.

Gaitan D y Pérez L. 2007. Aislamiento y Evaluación de Microorganismos celulolíticos a partir de residuos vegetales frescos y en compost generados en un cultivo de crisantemo (Dendranthema grandiflora). Tesis de Grado. Pontificia Universidad Javeriana, Facultad de Ciencia. Bogotá, Colombia.

Haichar FZ. 2007. Identification of cellulolytic bacteria in soil by stable isotope probing. Environ Microbiol, 9(3): 625-34.
Harrison C y Langdale J. 2006. Techniques for molecular analysis: A step by step guide to phylogeny reconstruction. The Plant Journal, 45: 561-572.

Heylen K, Lebbe L y De Vos P. 2008. Acidovorax caeni sp. nov., a denitrifying species with genetically diverse isolates from activated sludge. International Journal of Systematic and Evolutionary Microbiology, 58: 73-77.

Isla M, Craig A, Ordoñez R, Zampini C, Sayago J, Bedascarrasbure E, Alvarez A, Salomón V y Maldonado L. 2011. Physico chemical and bioactive properties of honeys from Northwestern Argentina LWT. Food Science and Technology, 44(9): 1922-1930.

Lane DJ. 1991. 16S/23S rRNA sequencing. In: Nucleic acid techniques in bacterial systematics. Stackebrandt, E., and Goodfellow, M., eds., John Wiley and Sons, New York, NY, pp. 115-175.

Lattaud C, Zhang BG, Locati S, Rouland C y Lavelle P. 1997. Activities of the digestive enzymes in the gut and in tissue culture of a tropical geophagous earthworm, Polypheretima elongata (Megascolecidae). Soil Biology and Biochemistry, 29(3): 335-339.

Miranda M, Leung K y Qin W. 2009. The prospects of cellulase-producing bacteria for the bioconversion of lignocellulosic biomass . International Journal of Biological Sciences, 5: 500-516.

Mitsuhiro U, Goto T, Nakazawa M, Miyatake K, Sakaguchi M y Inouye K. 2010. A novel cold-adapted cellulase complex from Eisenia foetida: Characterization of a multienzyme complex with carboxymethylcellulase, $\beta$-glucosidase, $\beta-1,3$ glucanase, and $\beta$-xylosidase. Comparative Biochemistry and Physiology Part B: Biochemistry and Molecular Biology, 157(1): 26-32. 
Nelson N. 1944. Photometric adaptation of the Somogyi method for thedetermination of glucose. The Journal of Biological Chemestry, 153: 375-38.

Prabha M L, Jayaraaj I y Jeyaraaj R. 2007. Comparative studies on the digestive enzymes in the gut of earthworms, Eudrilus eugeniae and Eisenia fetida eugeniae . Indian Journal of Biotechnology, 6: 567569.

Ramirez P y Coha J M. 2003. Degradación enzimática de celulosa por actinomicetos termófilos: aislamiento, caracterización y determinación de la actividad celulolítica. Revista Peruana de Biología, 10(1): 67-77.

Salvador C, Destain J, Rojas M y Paz-y-Miño C. 2011. Producción de actividades enzimáticas por bacterias del intestino de Eisenia foetida (Annelida: Clitellata: Haplotaxida). Revista Ciencia, 14(2): 193-199.

Sessitsch A, Coenye A, Sturz V, Vandamme P, Ait Barka E, Salles JF, Van Elsas JD, Faure D, Reiter B, Glick BR, Wang-Pruski G y Nowak J. 2005. Burkholderia phytofirmans sp. nov., a novel plant-associated bacterium with plant-beneficial properties International Journal of Systematic and Evolutionary Microbiology, 55: 1187-1192.
Turner S, Pryer KM, Miao VPW y Palmer, J. 1999. Investigating deep phylogenetic relationships among cyanobacteria and plastids by small subunit rRNA sequence analysis. Journal of Eukaryotic Microbiology, 46: 327-338.

Vinceslas-Akpa M y Loquet M. 1995. Observation in- situ de la microflore liée au tube digestif de Eisenia foetida andrei(Lumbricidae). European Journal of Soil Biology, 31(2): 101-110.

Zirbes L. 2007. Ecologie chimique d'Eisenia foetida et son implication dans le lombricompostage. Mémoire de fin d'études en vue de l'obtention du grade de Bioingénieur en chimique et Bioindustries. Faculté Universitaire des Sciences agronomiques Gembloux-Belgique. 